\title{
Video Article \\ In Vitro Microfluidic Disease Model to Study Whole Blood-Endothelial Interactions and Blood Clot Dynamics in Real-Time
}

\author{
Xue D. Manz ${ }^{1}$, Hugo J. Albers ${ }^{2,3}$, Petr Symersky ${ }^{4}$, Jurjan Aman ${ }^{1}$, Andries D. van der Meer ${ }^{3}$, Harm Jan Bogaard ${ }^{1}$, Robert Szulcek ${ }^{1,5,6}$ \\ ${ }^{1}$ Department of Pulmonary Diseases, Amsterdam UMC, VU University Medical Center, Amsterdam Cardiovascular Sciences (ACS) \\ ${ }^{2}$ BIOS Lab-on-a-Chip group, University of Twente \\ ${ }^{3}$ Applied Stem Cell Technologies Group, University of Twente \\ ${ }^{4}$ Department of Cardio-thoracic Surgery, Amsterdam UMC, VU University Medical Center \\ ${ }^{5}$ Institute of Physiology, Charité-Universitätsmedizin \\ ${ }^{6}$ German Heart Center
}

Correspondence to: Robert Szulcek at Robert.szulcek@charite.de

URL: https://www.jove.com/video/61068

DOI: doi:10.3791/61068

Keywords: Immunology and Infection, Issue 159, thrombosis, chronic thromboembolic pulmonary hypertension, endothelial cells, platelets, fibrin, coagulation, blood

Date Published: $5 / 24 / 2020$

Citation: Manz, X.D., Albers, H.J., Symersky, P., Aman, J., van der Meer, A.D., Bogaard, H.J., Szulcek, R. In Vitro Microfluidic Disease Model to Study Whole Blood-Endothelial Interactions and Blood Clot Dynamics in Real-Time. J. Vis. Exp. (159), e61068, doi:10.3791/61068 (2020).

\section{Abstract}

The formation of blood clots involves complex interactions between endothelial cells, their underlying matrix, various blood cells, and proteins. The endothelium is the primary source of many of the major hemostatic molecules that control platelet aggregation, coagulation, and fibrinolysis. Although the mechanism of thrombosis has been investigated for decades, in vitro studies mainly focus on situations of vascular damage where the subendothelial matrix gets exposed, or on interactions between cells with single blood components. Our method allows studying interactions between whole blood and an intact, confluent vascular cell network.

By utilizing primary human endothelial cells, this protocol provides the unique opportunity to study the influence of endothelial cells on thrombus dynamics and gives valuable insights into the pathophysiology of thrombotic disease. The use of custom-made microfluidic flow channels allows application of disease-specific vascular geometries and model specific morphological vascular changes. The development of a thrombus is recorded in real-time and quantitatively characterized by platelet adhesion and fibrin deposition. The effect of endothelial function in altered thrombus dynamics is determined by postanalysis through immunofluorescence staining of specific molecules.

The representative results describe the experimental setup, data collection, and data analysis. Depending on the research question, parameters for every section can be adjusted including cell type, shear rates, channel geometry, drug therapy, and postanalysis procedures. The protocol is validated by quantifying thrombus formation on the pulmonary artery endothelium of patients with chronic thromboembolic disease.

\section{Introduction}

The endothelium forms the inner cellular layer of blood vessels and separates blood from the surrounding tissue. It has been described as a dynamic organ that actively regulates its micro-environment and responds to external stimuli ${ }^{1}$. Because of its direct contact with flowing blood, the endothelium is pivotal in the control of hemostasis and thrombosis and is the primary source of many of the major regulatory molecules that control platelet aggregation, coagulation, and fibrinolysis ${ }^{2}$. Healthy, nonactivated endothelial cells (EC) produce several molecules that counteract platelet activation and prevent coagulation and thrombus formation to maintain blood flow, such as prostacyclin, thrombomodulin, or tissue factor pathway inhibitor (TFPI) ${ }^{2,3}$. This prevents the adhesion of platelets, platelet aggregation, and thrombus formation. Injury or activation of the vessel wall results in a procoagulant endothelial phenotype that initiates localized platelet adhesion and clot formation ${ }^{2,4}$. Upon endothelial activation platelets adhere to von Willebrand Factor (VWF), a multimeric protein released from ECs, or to exposed binding sites of the underlying subendothelial matrix. Subsequently, molecular changes in platelets and the exposure to tissue factor (TF) initiate the activation of the coagulation system, which induces thrombus formation by fibrin polymerization ${ }^{5,6}$. Together, the resulting clot provides the basis for wound closure by re-endothelialization ${ }^{7}$. Perturbations of the coagulation system may result in bleeding disorders, such as von Willebrand disease, hemophilia, or thrombosis, that often result from a dysregulated pro- and antithrombotic balance of the endothelial hemostatic pathway ${ }^{2,3}$.

The process of hemostasis occurs in both arterial and venous circulation. However, the mechanisms underlying arterial and venous thrombosis are fundamentally different. While arterial thrombosis, as seen in ischemic heart disease, is mostly driven by the rupture of an atherosclerotic plaque under conditions of high shear stress, venous thrombosis mostly develops in the absence of endothelial injury in a condition of stasis $^{8,9,10}$. A deep vein thrombus may embolize and travel towards the pulmonary arteries, where it causes a pulmonary embolism. This can result in chronic vascular obstructions leading to significant impaired functional capacities that might foster the development of chonic lung diseases including the development of chronic thromboembolic pulmonary hypertension (CTEPH) ${ }^{11,12,13,14}$. CTEPH is characterized by elevated pulmonary pressure due to obstructions of the pulmonary arteries by thromboembolic material following at least three months of anticoagulation therapy ${ }^{15}$. In addition to lung emboli, it is postulated that the pulmonary endothelium provides a prothrombotic environment in CTEPH that 
facilitates in situ thrombosis and chronic obstructions of the pulmonary arteries, causing the increase in blood pressure that ultimately can result in heart failure, if untreated ${ }^{16,17}$.

Over the past years, various studies have led to the development of assays to examine thrombus formation by measuring platelet function and coagulation ${ }^{18}$. However, most of them either study the interaction of whole blood with single extracellular matrix components like collagens or fibrins, or endothelial function in interaction with single blood components, such as endothelial-platelet or endothelial-leukocyte interaction ${ }^{19,20,21,22}$. These assays are most commonly performed with human umbilical vein endothelial cells (HUVEC), as these cells are easily obtained. However, hemostatic genes are differentially expressed across the vascular tree, vessel types, and organ systems ${ }^{23,24}$, which makes the use of HUVECs to represent endothelial cells involved in arterial thrombosis or pulmonary embolisms problematic ${ }^{23}$.

In addition to EC plasticity, disease-specific hemodynamic alterations and changes in vascular morphology can promote thrombus formation at the normal endothelium ${ }^{25}$. Higher shear rates, due to local vasoconstriction or changes in vessel geometry, for example, may result in acute thrombus formation, causing a stenosis that accelerates the cessation of blood flow ${ }^{26}$. The use of custom-made microengineered flow channels allows to specifically design vascular geometries that are representative of the (patho)biology. In this way, it is possible to study the effect of local biomechanical forces on healthy or diseased $\mathrm{EC}^{27}$.

There are anticoagulation therapies available for targeting different phases and molecules in the coagulation cascade, which all pose particular risks and benefits that can be specific to certain disorders. The approach of disease modeling described in this paper is especially suited to test the effects of various anticoagulation and antiplatelet therapies on thrombus dynamics.

The aim is to present a model of thrombosis that includes primary ECs, yielding a versatile model suitable for the analysis of various forms of thrombosis depending on the type of primary ECs used. As an illustration, we used pulmonary artery endothelial cells from CTEPH patients in interaction with whole human blood containing all components involved in thrombus formation (platelets, leukocytes, erythrocytes, clotting proteins, and cofactors). This approach can be applied in commercial parallel flow channels or in custom-made microfluidic flow channels with a specific vascular design. As such, the model can eventually be used in the study of thrombus formation and resolution, for the assessment of inflammatory responses in disease modeling, for antiplatelet or anticoagulation therapy, and ultimately for personalized medicine.

This study describes the isolation of primary human pulmonary artery endothelial cells. For the isolation of other primary human endothelial cell types, we refer to previously published methods, including pulmonary microvascular endothelial cells ${ }^{25}$, human umbilical vein endothelial cells ${ }^{28}$, and blood circulating endothelial colony forming cells Figure $1 \mathrm{~A}^{29}$.

\section{Protocol}

This study was approved by the institutional Medical Ethical Review Board of the VU Medical Center Amsterdam, The Netherlands (METC VUmc, NL69167.029.19). Primary cell isolation and blood collection of human subjects was performed after written informed consent was obtained in accordance with the Declaration of Helsinki.

\section{Isolation and culture of primary human pulmonary arterial endothelial cells (PAEC)}

1. Warm complete endothelial cell medium (cECM) supplemented with $5 \%$ fetal bovine serum (FBS), $1 \%$ penicillin/streptomycin (P/S), $1 \%$ endothelial cell growth supplements (ECGS), and $1 \%$ nonessential amino acids (NEAA), in a water bath at $37^{\circ} \mathrm{C}$. Sterilize surgical scissors, forceps, and a scalpel with either a $120^{\circ} \mathrm{C}$ heat sterilizer or $70 \%$ ethanol. Perform the isolation of PAEC in a laminar flow cabinet under sterile conditions.

2. Coat a high affinity cell binding $60 \mathrm{~mm}$ cell culture dish (see Table of Materials) with $2 \mathrm{~mL}$ of $5 \mu \mathrm{g} / \mathrm{mL}$ fibronectin and incubate at $37{ }^{\circ} \mathrm{C}$ for at least $15 \mathrm{~min}$.

3. Obtain pulmonary artery (PA) tissue from surgery (e.g., lobectomy or pulmonary endarterectomy), store in $4{ }^{\circ} \mathrm{C}$ cold cord buffer $(4 \mathrm{mM}$ potassium chloride, $140 \mathrm{mM}$ sodium chloride, $10 \mathrm{mM} \mathrm{HEPES}, 11 \mathrm{mM}$ D-glucose, and $1 \% \mathrm{P} / \mathrm{S}, \mathrm{pH}=7.3$ ), and keep on ice until isolation.

4. Isolate endothelial cells from PA within $2 \mathrm{~h}$ after tissue removal. Take the PA with forceps and put it in a $10 \mathrm{~cm}$ Petri dish to wash with PBS. If the PA is still a ring, cut the pulmonary artery open with scissors. Be very careful to not touch the innermost layer of the vessel with the tools, as the endothelium is easily damaged and/or removed.

5. Remove the fibronectin from the cell culture dish and add $4 \mathrm{~mL}$ of $\mathrm{cECM}$ medium.

6. Take the PA tissue with forceps and place it in the medium. Meanwhile, carefully scrape the inner layer of the vessel into the medium with a scalpel. Often, lipid accumulations can be seen in the vessel wall. Try to omit these, as they will impact cell outgrowth.

7. Keep the cells in culture until small colonies of endothelial cells start to appear.

8. Replace the medium every other day. If fibroblasts contaminate the culture, purify it with magnetic affinity cell separation for CD144 with a kit, in accordance to the manufacturer's instructions (see Table of Materials). Use the two-column method for the initial purification and the onecolumn method for every consecutive purification. Generally, a culture is defined pure when flow cytometry detects $\leq 10 \%$ contaminating cells.

9. Split cells in ratios of 1:3 to 1:4 until sufficient PAECs are grown for experimental use. When PAECs are ready to use, continue with the next step.

10. After purification, primary endothelial cells need to be characterized for the presence of EC specific markers (e.g., VE-cadherin, CD31, TIE2) and for the absence of smooth muscle cells ( $\alpha-S M A)$, fibroblast (vimentin), and epithelial (cytokeratin) markers (Figure 1B).

\section{Preparation of flow chambers and PAEC monolayers}

NOTE: Depending on the hypothesis, use either the commercially available flow chambers (see Table of Materials and Figure 1C Option A, step 2.1 of the protocol) or a custom-made microfluidic flow chamber (Figure 1C Option B, step 2.2 of the protocol).

1. Cell seeding of endothelial monolayers in commercially available microslides 
NOTE: Commercially available flow chambers are easy to use and provide a laminar flow pattern throughout the channel that can be used at high shear rates. These microslides are designed to allow multichannel parallel runs and provide an accurate and reproducible flow profile. Because they are optimized for inverted microscopy, it is possible to capture high quality fluorescent images. Furthermore, various dimensions of flow chambers are available in different channel sizes or geometries. The 6-well flow channels are preferred for this assay because these microslides have small dimensions and allow multiparallel runs.

1. To coat one channel of a 6 -well flow slide ( $3.5 \mathrm{~mm}$ width $\times 0.4 \mathrm{~mm}$ height $\times 17 \mathrm{~mm}$ length) use $30 \mu \mathrm{L}$ of $0.1 \%$ gelatin per channel and incubate at $37^{\circ} \mathrm{C}$ for at least $15 \mathrm{~min}$.

2. To seed one channel, trypsinize $10 \mathrm{~cm}^{2}$ of confluent PAECs and spin down at $300 \mathrm{xg}$ for $7 \mathrm{~min}$ at room temperature (RT).

3. Suspend PAECs in $600 \mu \mathrm{L}$ of cECM and pipette $100 \mu \mathrm{L}\left(1.5 \mathrm{~cm}^{2}\right)$ of cell suspension in one channel. This covers the microslide through capillary action. If it is going too slow, air bubbles will form. Avoid the formation of air bubbles by using a little bit more force when pipetting.

NOTE: Cell density influences endothelial phenotype. A total of $1.5 \mathrm{~cm}^{2}$ of confluent cells per channel is overconfluent, because the channel has a surface area of $0.6 \mathrm{~cm}^{2}$. Before starting the experiment, culture these cells for another 6 days within the channels until they reach maximum confluency.

4. Add an additional $50 \mu \mathrm{L}$ of $\mathrm{cECM}$ to the channel to provide sufficient medium for overnight incubation at $37{ }^{\circ} \mathrm{C}, 5 \% \mathrm{CO}_{2}$. $\mathrm{Change}$ the medium the next day to wash away unbound cells.

5. Keep the cells in culture for 6 days at $37{ }^{\circ} \mathrm{C}, 5 \% \mathrm{CO}_{2}$ to allow the PAEC to form a firm, confluent monolayer. Change the medium every other day with $150 \mu \mathrm{L}$ of $\mathrm{CECM}$.

6. At day 7 , treat the PAEC with $100 \mu \mathrm{L}$ of $1 \mu \mathrm{M}$ histamine in ECM and $1 \%$ FBS without any other additives for 30 min prior to the assay. The stimulus can be chosen ad libitum. For example, TNF- $\alpha$ has been commonly used as an inflammatory activator.

2. Preparation of custom-made microfluidic flow chambers

NOTE: Custom-made flow chambers are adapted to the user's needs because the dimensions and geometries can be easily changed to preference. For example, to mimic a more physiologically relevant vessel, a stenosis can be introduced (Figure 1D). This approach allows the use of very small blood volumes as seen in microvascular disease.

1. Soft lithography of polydimethylsiloxane (PDMS) using patterned wafers

1. Combine PDMS curing agent and prepolymer on a microbalance at a 1:10 ratio and thoroughly mix with a general purpose lab mixer.

2. To remove the resulting air bubbles, degas the PDMS in a desiccator for approximately $1 \mathrm{~h}$.

3. Line a glass Petri dish with aluminum foil and add a few droplets of water before placing the wafer in the lined Petri dish. The wafer serves as the mold for the custom-made channel.

NOTE: The wafers or molds are provided by the clean room facilities. Negative photoresist is patterned onto wafers to create protruding channel-like features with the following typical dimensions: $300 \mu \mathrm{m}$ width $\times 270 \mu \mathrm{m}$ height $\times 14 \mathrm{~mm}$ length.

4. Pour the degassed PDMS onto the wafer placed in a glass Petri dish.

5. Degas the PDMS poured onto the wafer in a desiccator for an additional $15 \mathrm{~min}$ to remove any bubbles that might have formed during casting.

6. Remove the Petri dish containing the mold and PDMS from the desiccator and place it in a $60{ }^{\circ} \mathrm{C}$ oven for a minimum of $4 \mathrm{~h}$ to cross-link the PDMS.

2. Preparation of cross-linked PDMS for bonding to glass slides

1. Remove mold and cross-linked PDMS from the oven and move to a cross-flow hood for dust-free handling of the PDMS.

2. Remove any PDMS from the bottom of the mold using a scalpel and remove the top slab of the cross-linked PDMS from the mold.

3. Line the exposed patterned PDMS slab with adhesive tape to prevent any dust particles to come into contact with the PDMS channels.

4. Cut the PDMS chips to size and punch $1 \mathrm{~mm}$ diameter inlets and outlets using a biopsy punch.

3. Sterilization and bonding of PDMS microfluidic channels and glass slides

1. Clean glass microscopy slides by thoroughly rinsing with ethanol followed by an isopropyl alcohol rinse. After each rinse, thoroughly dry the slides with a nitrogen gun. NOTE: Alternatively, cover slips can be used if the analysis is conducted using a confocal microscope.

2. Open the plasma chamber and load the cleaned microscopy slides and microfluidic chips without the protective tape.

3. Close the chamber, purge with nitrogen for $1 \mathrm{~min}$, and fill the chamber with filtered air until a pressure of $500 \mathrm{mTorr}$ is reached.

4. Expose the glass slides and PDMS chips to the plasma for $40 \mathrm{~s}$ using a power of $50 \mathrm{~W}$ and a frequency of $5 \mathrm{kHz}$.

5. After exposure to the plasma, immediately bond the glass slides and microfluidic chips. Store the devices in sterile Petri dishes.

3. Cell seeding of endothelial monolayers in microfluidic channels

1. Coat the custom-made microchannel by pipetting $10 \mu \mathrm{L}$ of $0.1 \mathrm{mg} / \mathrm{mL}$ collagen Type I or $0.1 \%$ gelatin per channel and incubate at 37 ${ }^{\circ} \mathrm{C}$ for $30 \mathrm{~min}$.

2. To seed four microchannels, trypsinize $25 \mathrm{~cm}^{2}$ of confluent PAECs and spin down at $300 \times g$ for $7 \mathrm{~min}$ at RT. NOTE: Only a small percentage of cells will adhere to the surface. Therefore, it is necessary to use an excess of cells to achieve a confluent monolayer.

3. Suspend PAECs in $20 \mu \mathrm{L}$ of cECM and use $5 \mu \mathrm{L}$ of cell suspension for each channel. Because of the small channel dimensions, the capillary forces will fill the microslide and prevent air bubble formation.

4. Change medium after $3-4 \mathrm{~h}$ to wash unbound cells.

5. Keep the cells in culture for 6 days to allow the PAEC to form a firm, confluent monolayer. Because of the small volume, change the medium every day with $150 \mu \mathrm{L}$ of cECM. Leave the pipette tip filled with medium in the inlet and put an empty tip in the outlet. This will serve as a reservoir providing sufficient nutrients and growth factors to the cells and prevent the slide from drying out.

6. On day 7 , stain the nuclei in the live cells with Hoechst $(1: 5,000$ in $\mathrm{CECM})$ and incubate for a maximum of 10 min at $37{ }^{\circ} \mathrm{C}$ and $5 \% \mathrm{CO}_{2}$. 
7. Carefully wash $3 x$ with $\mathrm{CECM}$ and treat with $1 \mu \mathrm{M}$ histamine in $\mathrm{ECM}+1 \% \mathrm{FBS}$ for 30 min prior to the assay. The stimulus can be chosen ad libitum. For example, TNF- $\alpha$ has been commonly used as an inflammatory activator.

8. Use $1.27 \mathrm{~mm}$ diameter $90^{\circ}$ angled stainless steel connectors to connect the outlet of the PDMS chips to tubing.

\section{Preparation of human whole blood}

1. Draw venous blood from subjects in $0.109 \mathrm{M}$ sodium citrate anticoagulant. This can be from healthy or diseased subjects that do not receive anticoagulation treatment. Gently invert the tubes to mix. The total amount of blood required for an experiment mainly depends on the selected flow rate. With a flow rate of $25 \mathrm{~mL} / \mathrm{h}$, in ibidi 6-well slides, a total volume of $2 \mathrm{~mL}$ with a little excess to fill up tubs and flow channel is sufficient.

2. Transfer the blood to a $50 \mathrm{~mL}$ tube and add Calcein $\mathrm{AM}(1: 10,000)$ to fluorescently label blood cells and Alexa488-fibrinogen (15 $\mu \mathrm{g} / \mathrm{mL})$ to conjugate autologous fibrinogen. Let the blood incubate at $37^{\circ} \mathrm{C}$ for $15 \mathrm{~min}$ to allow complete absorption.

3. Dilute the blood 1:1 with recalcification buffer ( $154 \mathrm{mM}$ sodium chloride, $10.8 \mathrm{mM}$ trisodium citrate, $2.5 \mathrm{mM}$ calcium chloride, and $2 \mathrm{mM}$ magnesium chloride) immediately before the start of experiment.

NOTE: Hemodilution with a maximum of $50 \%$ did not influence coagulation reaction time ${ }^{30}$. Furthermore, human blood can contain viruses and other agents. Working with blood samples, therefore, carries a risk of infection. It is highly recommended to use appropriate safety measures and to handle the material with care.

\section{Assembling the flow system}

1. Before connecting the tubing, rinse the flow tubes with a $20 \mathrm{~mL}$ syringe filled with wash buffer ( $36 \mathrm{mM}$ citric acid, $103 \mathrm{mM}$ sodium chloride, 5 $\mathrm{mM}$ potassium chloride, $5 \mathrm{mM}$ EDTA, and $0.35 \% \mathrm{wt} / \mathrm{vol}$ bovine serum albumin $[\mathrm{BSA}], \mathrm{pH}=6.5$ ) to prevent clotting of the blood in the tubes.

2. Use a new syringe to fill the flow tubes with HEPES buffer (132 mM sodium chloride, $20 \mathrm{mM}$ HEPES, $6 \mathrm{mM}$ potassium chloride, $1 \mathrm{mM}$ magnesium chloride, $1 \% \mathrm{BSA}$, and $5.5 \mathrm{mM} \mathrm{D}$-glucose, $\mathrm{pH}=7.4$ ) and carefully connect it with an elbow-shaped Luer connector to the microslide filled with EC in medium. While attaching the connectors to the microchannels, try to prevent the formation of any air bubbles in the slide. Bubbles will damage the endothelium and influence the results of your experiment. Remove wash buffer completely and don't let the buffer get in contact with the cells, as this will inhibit coagulation.

3. Set up the flow system as shown in Figure 1E. Take up $2 \mathrm{~mL}$ of wash buffer in a $20 \mathrm{~mL}$ syringe and rinse to prevent clotting when the blood enters the syringe. Insert the empty syringe in the syringe pump and connect the outlet tube with a female Luer connector to this syringe. Put the inlet tube in a container containing the prepared human blood.

4. Switch on the syringe pump and calculate the flow rate. Flow profiles follow a parabolic pattern in height. Assuming that the blood acts as a Newtonian fluid, use the following formula to calculate flow rate.

$\tau=\eta \frac{6 Q}{h^{2} w} \quad$ (Equation 1)

where

$\mathrm{T}=$ shear stress $\left[\frac{\mathrm{dyn}}{\mathrm{cm}^{2}}\right]$

$\eta=$ dynamical viscosity $\left[\frac{d y n \times s}{\mathrm{~cm}^{2}}\right]$

$\mathrm{Q}=$ volumetric flow rate $\left[\frac{\mathrm{mm}^{\mathrm{s}}}{\mathrm{s}}\right]$

$\mathrm{h}=$ channel height $[\mathrm{mm}]$

$\mathrm{w}=$ channel width $[\mathrm{mm}]$

NOTE: For pulmonary arterial flow with blood in the commercial 6 channel microslides with rectangular dimensions with $0.4 \mathrm{~mm}$ height and $3.5 \mathrm{~mm}$ width, a volumetric flow rate of $25 \mathrm{~mL} / \mathrm{h}$ was used for $5 \mathrm{~min}$. Due to the differences in the dimensions of the custom-made flow channels, these dynamics will also change. Adjust the variables to make sure the shear stress is equal.

5. Define the diameter of the $20 \mathrm{~mL}$ syringe to $19.05 \mathrm{~mm}$ and set the program to Withdraw. Pulling the blood through the cell-coated channel by application of a negative pressure will guarantee constant shear rates and minimal damage to the cell layer.

\section{Setting up the microscope for image acquisition}

1. Use a 20x objective and place the microslide onto the stage of the inverted phase contrast fluorescent microscope. Start the microscope software to move the stage in the Z-direction to focus on the cell monolayer.

2. Select a region of interest (ROI) in the beginning, middle, and end of every flow channel. The beginning and end should be at least $3 \mathrm{~mm}$ away from the inlet and outlet of the channel. Set the blue, green, and red fluorescent filters with a laser power and light intensity that shows minimal background.

\section{Perfusion of whole blood over PAECs}

1. After everything is connected as in Figure $\mathbf{1 E}$ and the microscope is ready for recording, push Start to record a video. Push Start on the syringe pump to perfuse the blood over the endothelium.

2. As soon as the blood starts flowing over the endothelium, acquire images with the preselected active channels and ROI positions every $15 \mathrm{~s}$ for 5 min.

3. After 5 min of perfusion, finish recording and stop the syringe pump. Disassemble the flow chamber and very carefully remove the tubing. Often, an air bubble will form if this is done with too much force. Try to prevent this, because this will flush away the endothelium. 


\section{Fixation and post-analysis}

NOTE: To exclude false positive platelet adhesion by endothelial damage due to the perfusion experiment, it is necessary to characterize the endothelial cells for their gap formation and monolayer. This can be done by regular immunofluorescence staining for VE-cadherin.

1. After perfusion and disassembling of the tubing, wash the microfluidic channel with HEPES. Pipette HEPES into the channel so that it will push the blood to the outlet. Remove the excess with another pipette.

1. Optionally, wash the channel with $\mathrm{PBS}^{++}$(with $0.5 \mathrm{mM}$ magnesium chloride and $0.9 \mathrm{mM}$ calcium chloride) to prevent precipitation of protein supernatant. This reduces background. However, an extra washing step can change cellular behavior and morphology that could influence post-analysis imaging.

2. Remove the HEPES and fix the endothelium with adhered platelets and deposited fibrin by pipetting $37{ }^{\circ} \mathrm{C}$ warmed $4 \%$ paraformaldehyde (PFA) into the channel and incubate for $15 \mathrm{~min}$ at RT.

NOTE: Be extra careful when fixing the custom-made microfluidics as these channels are even smaller, which causes higher shear forces in the channel during every handling step.

3. Remove the PFA from the channel and wash $3 x$ with PBS. The microslides are now ready for a standard staining protocol to characterize endothelial cell markers such as VE-cadherin, CD31, P-selectin or integrins, CD42b for adherent platelets, or CD45 for leukocytes.

4. After staining, take at least five images with a regular confocal microscope to characterize colocalization.

\section{Image analysis}

1. Open a flow assay image containing either fluorescently labeled platelets or fluorescent fibrin in ImageJ. Drag image of choice to ImageJ.

2. The image is taken in RGB color. For analysis, an 8-bit image is preferred, so transform the image into 8-bit: Image | Type | 8-bit.

3. To minimize the background, subtract with a sliding paraboloid, where a rolling ball locally calculates and subtracts background pixels from the original image: Process | Subtract Background | Rolling Ball Radius $=\mathbf{5 0}$ pixels | Sliding Paraboloid.

NOTE: The image size is defined in pixels. However, to measure area, it is necessary to set the scale. When the images are taken with a 20x objective with a numerical aperture of 0.45 , the microscope has a scale of 2 pixels per $\mu \mathrm{m}$. Most of the time the necessary information to set the scale is stored in the metadata of the image and can automatically be used by ImageJ: Analyze | Set Scale.

4. Set a threshold to define adhered platelets or deposited fibrin. Use the Triangle method and the threshold will adjust automatically: Image | Adjust | Threshold.

5. Analyze the area covered by the platelets or fibrin with the Analyze Particles command. Set size at 2-Infinity, as the minimal size of a platelet is $2 \mu \mathrm{m}$ : Analyze | Analyze Particles.

6. The results will provide the total area in $\mu \mathrm{m}^{2}$, with average size of the aggregates in $\mu \mathrm{m}^{2}$ and the percentage of covered area.

\section{Representative Results}

The representative results can be divided into three parts, each representing the respective steps of experimental setup, data collection, and data analysis. Depending on the research question, parameters for each step can be changed. The presented data are applied to study the influence of the endothelium on thrombus formation.

\section{Experimental Setup}

It is well-established that endothelial cells are highly heterogeneous in structure and function, depending on location and time, during health and disease $^{23}$. Various sources of endothelial cells can be used to study endothelial-blood interaction, which in this case were commercially available HUVECs and PAECs (Figure 1A). HUVECs have been the most commonly used in laboratory models, while PAECs are patient-derived isolated cells from the pulmonary arteries. Furthermore, there are well-established protocols available to isolate microvascular endothelial cells (MVEC) from the pulmonary circulation or blood circulating endothelial colony forming cells $\left(\right.$ ECFC) ${ }^{25,28,29}$.

After isolation, endothelial cells were characterized by VE-cadherin, CD31, and TIE2 staining to confirm an endothelial phenotype.

Characterization for the presence of aSMA and cytokeratin indicated the absence of a fibroblast or epithelial-like phenotype (Figure 1B). After obtaining a highly pure population of ECs, passage 3-5 cells were used to seed either a commercial microslide or custom-made microfluidic flow channels (Figure 1C). While the commercially available microslides are primarily parallel flow chambers, or Y-shaped channels with specifically defined parameters in height or bifurcation angle, the use of microfluidic slides makes it possible to adapt the experimental parameters to in vivo vessel geometry and blood flow dynamics ${ }^{31}$. However, custom-made microfluidic sizes are smaller and tend to limit cell spread and induce more cell death ${ }^{32,33}$. Using a surplus of cells compensates for the fact that only a small percentage of cells will adhere. This was observed when a stenosis was introduced, where endothelial cells showed a more elongated phenotype compared to a parallel microfluidic channel (Figure 1D). Commercial flow chambers have a bigger surface area that cells can bind to. This requires fewer cell numbers for seeding.

To study endothelial cell-blood interaction, whole blood was perfused over an endothelial monolayer. Citrated blood was collected on the day of the experiment and immediately before perfusion recalcified. Cells were stimulated with histamine to induce VWF release and platelet adhesion 30 min prior to perfusion (Figure 1E) ${ }^{34,35}$. Because of the small dimensions, the custom-made microfluidic channels allowed use of smaller blood volumes.

\section{Data collection}

To investigate thrombus formation on PAECs, Calcein AM-Red fluorescently labeled blood cells and Alexa488-conjugated fibrin were perfused for $5 \mathrm{~min}$ at $2.5 \mathrm{dyne} / \mathrm{cm}^{2}$ (Figure 2A-C). Adherent blood cells and deposited fibrin were quantified. Images were acquired every $30 \mathrm{~s}$ and quantified with ImageJ. It was important to subtract the background to eliminate the autofluorescence of nonadhered platelets. The triangle algorithm for thresholding has been used to define minimal background. It allowed for measurement of small platelet aggregates (Figure 2D). 
Expectedly, under nonstimulated conditions, there was no binding of platelets and fibrin to the endothelium. To promote VWF release and platelet binding, PAECs were stimulated with histamine, which resulted in an immediate increase of platelet adhesion reaching a plateau after 2.5 min. At this time, platelets started to secrete autocrine factors that induced platelet aggregation and fibrinogen cleavage into fibrin. Fibrin was deposited after $3 \mathrm{~min}$ and formed a stable aggregate with platelets after 4 min (Figure 2E).

To investigate whether this effect could be inhibited by a direct oral anticoagulant (DOAC), blood was treated with $10 \mathrm{nM}$ dabigatran. Dabigatran was added to the blood dilution, where it inhibits Factor lla in the coagulation pathway, and prevented the cleavage of fibrinogen to form fibrin fibers. When dabigatran-treated blood was perfused over stimulated PAECs, clot formation could be directly inhibited mainly by delaying fibrin deposition (Figure 2F).

\section{Data analysis}

To study the influence of various endothelial sources on thrombus formation, the cellular changes upon 5 min blood perfusion were analyzed. The endothelium was fixed and adherent platelets were labeled with CD42b before imaging under a confocal microscope. This provided a detailed analysis for colocalization of platelets and fibrin that could indicate dysfunctional coagulation factors in the blood. The influence of $\mathrm{EC}$ in clot formation was determined by standard immunofluorescent staining. Endothelial cell-cell contacts were maintained, as confirmed by VE-cadherin staining,indicating that the blood clots formed on top of the endothelial monolayer rather than on the underlying matrix in between endothelial gaps (Figure 3). Furthermore, the use of different cell sources resulted in different patterns of thrombi forming on the endothelium. HUVECs are venous endothelial cells and showed limited platelet adhesion and fibrin deposition, while diseased primary PAEC from CTEPH patients showed abundant platelet adhesion and more fibrin deposition upon histamine stimulation compared to healthy PAEC. This suggests that the endothelium of CTEPH patients show higher responsiveness to vasoactivation that results in increased thrombus formation.

\section{A}
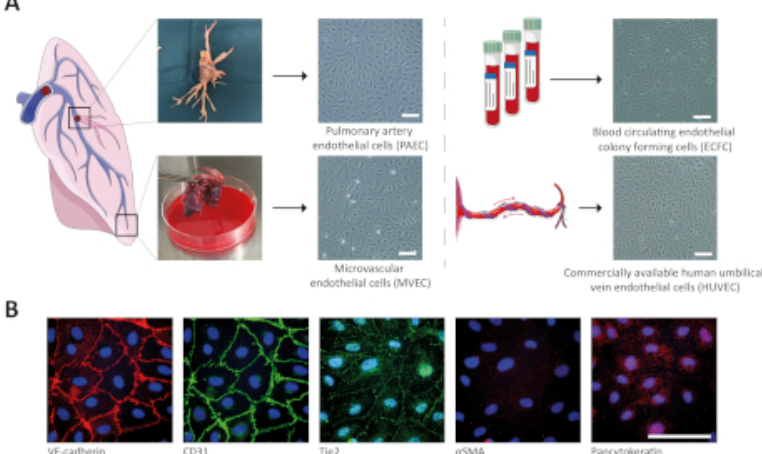

C

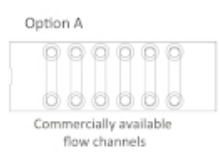

OR

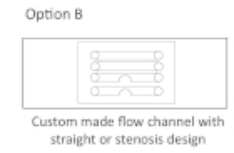

D

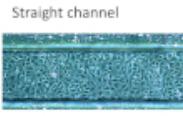

OR

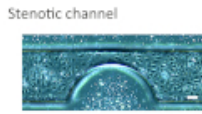

E

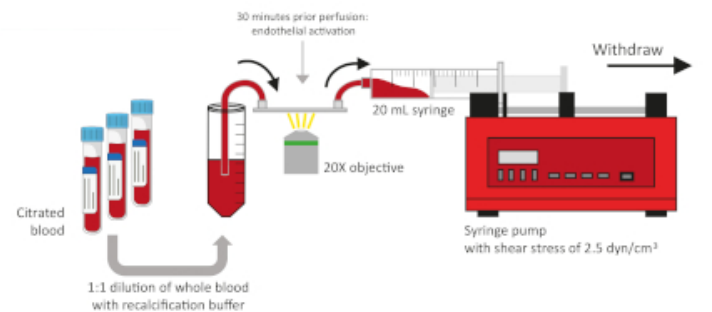

Figure 1: Schematic overview of the protocol. (A) Various sources and different types of endothelial cells were isolated and cultured for usage in a microfluidic flow channel for perfusion experiments. Representative brightfield images of different types of endothelial cells. Scale bar = $50 \mu \mathrm{m}$. (B) Isolated cells were characterized by immunofluorescent staining to confirm an endothelial phenotype. Scale bar $=50 \mu \mathrm{m}$. (C) Cells can be seeded in either commercial flow slides or custom-made microfluidic channel. (D) Representative brightfield images of HUVEC grown in different channel geometries. Scale bar $=50 \mu \mathrm{m}$. (E) Experimental setup of blood perfusion experiments. Citrated blood was collected, diluted with saline buffer, and perfused over endothelial cells with a syringe pump. The lung and umbilical vein in this figure were modified from Servier Medical Art, licensed under a Creative Common Attribution 3.0 Generic Licence. http://smart.servier.com/ Please click here to view a larger version of this figure. 
A Unstimulated PAEC

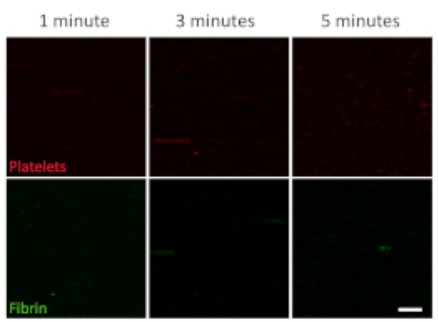

C Histamine stimulated PAEC with Dabigatran 1 minute
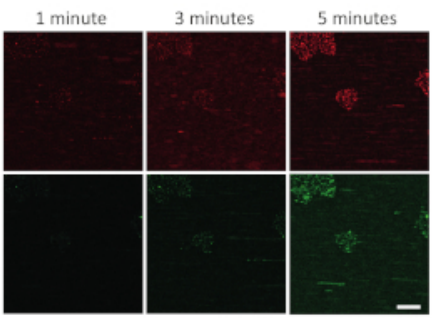

B Histamine stimulated PAEC

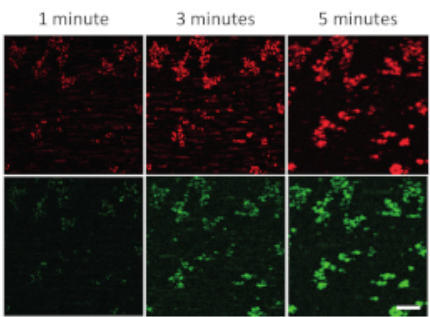

D

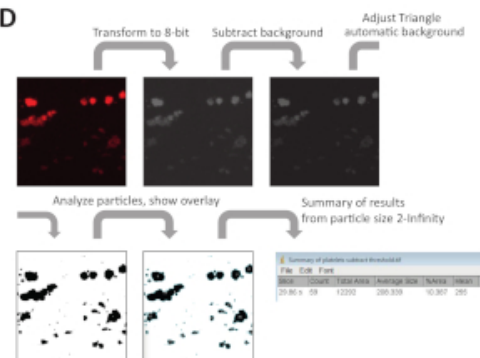

F
Platelet adhesion

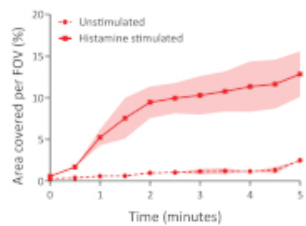

Fibrin deposition

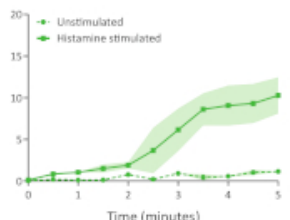

Dabigatran treatment

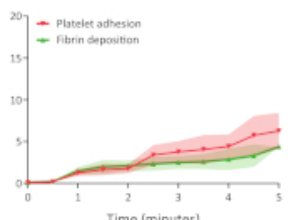

Figure 2: Image acquisition and quantification of thrombus formation. (A) Representative time-lapse imaging of adhered Calcein AM-Red labeled platelets and deposited Alexa488-conjugated fibrin at 1, 3, and 5 min after whole blood perfusion over unstimulated PAECs (B) and over histamine stimulated PAECs. (C) Representative time-lapse images of platelet adhesion and fibrin deposition at 1, 3, and 5 min of perfusion with whole blood incubated with dabigatran perfused over histamine-stimulated PAECs. Scale bar $=50 \mu \mathrm{m}$. (D) Schematic overview of image quantification in ImageJ. (E) Quantification of platelet adhesion and fibrin deposition for every $30 \mathrm{~s}$ on an unstimulated and histamine stimulated endothelium. (F) Quantification of the effect of dabigatran on thrombus formation quantified by platelet adhesion and fibrin deposition. Data are represented as mean $\pm S D, n=3$. Please click here to view a larger version of this figure. 


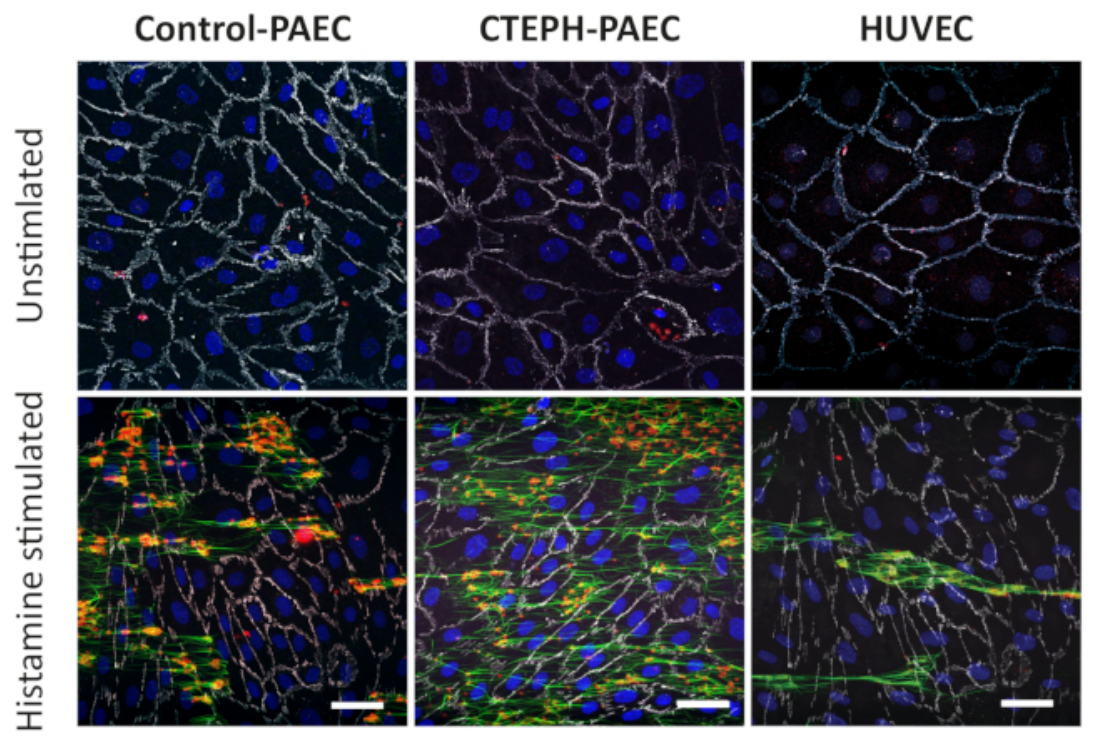

Figure 3: Representative confocal images of flow experiments to characterize thrombus formation in platelet adhesion and fibrin deposition on endothelial cells. Endothelial cell-cell contacts were characterized by VE-cadherin and measured in control PAECs, patientderived CTEPH PAECs, and HUVEC. Scale bar $=50 \mu \mathrm{m}$. Please click here to view a larger version of this figure.

\section{Discussion}

Coagulation is a result of the complex and temporally controlled interplay between the endothelium and blood components. This in vitro assay presents a method to investigate thrombogenic properties of endothelial cells in real-time. Various types of primary human endothelial cells can be used, facilitating in situ thrombosis in an organ and patient-specific manner. In this study, we illustrated the use of this protocol comparing thrombogenic properties of PAECs isolated from healthy donors versus CTEPH patients. Live thrombus formation was studied by perfusion of whole blood from healthy subjects over histamine-activated endothelium, while the effect of a DOAC was tested as an antithrombotic agent.

Besides the use of commercially available microchannels, as shown in the representative results, the introduction of the custom-made microfluidic channels enables the study of the influence of vascular geometry changes on thrombus formation. For example, flow decreases at a branch point or stenosis results in an increase in shear stress and more platelet activation ${ }^{36}$. However, a significant limitation of these custommade microfluidic channels is the requirement of high cell numbers to form a stable monolayer of ECs as described in step 2.3.2. This may present a limiting factor when patient-derived cells are scarce. The strengths of the commercially available microslides are that surface areas are modified for cell culture and growth areas are bigger, thereby allowing ECs to form a confluent and stable monolayer. On the other hand, a bigger surface area will result in a bigger lumen. According to Equation 1, this requires higher blood volumes to reach similar flow rates as in the custom-made microfluidics.

An improvement to this protocol could be to investigate live EC loss or changes in barrier integrity. EC damage in this protocol is only measured at the end of the experiment. For live tracking, ECs can be tagged with mCherry VE-cadherin, for example ${ }^{37}$. However, as this would need a highly optimized protocol with efficient virus transfection, Electric Cell-substrate Impedance Sensing (ECIS) could be used as an alternative to study endothelial integrity and barrier function ${ }^{38}$. Perfusion over special ECIS flow channels allows longitudinal monitoring of endothelial barrier integrity under flow. These specific ECIS features allow for parallel measurements of endothelial barrier properties and thrombus formation. Alternative ways for parallel EC barrier measurements, especially in the custom-made arrays include the use of fluorescent dextrans in the perfusate, which diffuse out of the lumen, depending on the EC barrier properties.

A limitation of the described protocol is that ECs are removed from the human body and cultured on tissue culture plastic, which is a stiff, artificial substrate. Cells adapt to their biophysical environment. This could possibly affect endothelial response to platelet activation, as there is an association between platelet activation and wall stiffness ${ }^{39}$. Despite these adaptations to culture plastics, cells do keep disease-specific characteristics that can be identified in direct comparison with ECs derived from healthy donors as shown with control, CTEPH-PAEC, and HUVEC that exerted different patterns of platelet adhesion and fibrin deposition after 5 min of blood perfusion.

In contrast to other protocols, this system uses whole blood while others study EC interaction with a single blood component such as platelets and leukocytes ${ }^{19,20,21}$. There have been more advanced microfluidic models developed that allow the study of endothelial function in a vascular model with a round vessel geometry and soft extracellular matrix. However, these are optimized with HUVECs ${ }^{40,41,42}$. The novelty of the described protocol is the use of primary ECs combined with whole blood bringing the modelling of in situ thrombosis one step closer to in vivo conditions. Having optimized the protocol for the use of patient-derived ECs and patient-derived blood further optimizes disease modelling in vitro, allowing the assessment of personalized thrombus formation and drug treatment.

The described protocol can be applied to study the effect of anticoagulation therapies on patient-derived cells. While we used dabigatran to inhibit thrombus formation, it is possible to use other anticoagulants, such as rivaroxaban, which directly inhibits factor Xa in the coagulation cascade. Direct-platelet inhibitors like clopidogrel or aspirin can be studied as well, as these act on the primary phase of hemostasis where 
platelets bind to ECs. Ultimately, the thrombogenic capacities of patient-specific ECs in interaction with the patient's own blood can be used to predict the personal effect of anticoagulation therapy on the individual patient. Furthermore, a knockdown of specific proteins can provide additional functional information.

There are some steps in the described protocol that are critical for a successful perfusion experiment. First, during the isolation of primary cells, it is necessary to obtain a highly pure EC culture. Second, it is important that the ECs form a stable confluent monolayer. If this is not the case, a slight change in shear stress can cause endothelial damage and activation of the coagulation cascade, or platelets can start binding to the basement membrane, which will provide false positive results. Third, it is essential to prevent air bubbles, as those can damage the endothelium and thereby influence the results.

After recalcification of the citrated blood with calcium chloride and magnesium chloride, it is important to immediately start the perfusion experiment. Recalcification induces a rapid response in platelet activation and thrombus formation, resulting in fast clotting in the sample.

In conclusion, we describe a highly versatile protocol to study whole blood-endothelial cell interactions during thrombosis.

\section{Disclosures}

The authors declare no conflict of interest.

\section{Acknowledgments}

We thank Jan Voorberg from the Department of Plasma Proteins, Sanquin Research and Landsteiner Laboratory, Amsterdam UMC, Academic Medical Center, Amsterdam, The Netherlands, for his input in this manuscript. This work was supported by the Dutch CardioVascular Alliance (DCVA) [2012-08, 2014-11] awarded to the Phaedra and the Reconnect consortium as well as the Impulse grant 2018 awarded to the Phaedra IMPACT consortium. These grants include collective funding by the Dutch Heart Foundation, Dutch Federation of University Medical Centers, The Netherlands Organization for Health Research and Development, and the Royal Netherlands Academy of Sciences. Furthermore, this work was funded by the European Research Council under the Advanced Grant 'VESCEL' program (Grant number: 669768). XDM is funded by a research grant of the Institute for CardioVascular Research (ICaR-VU) at the VU University Medical Center, Amsterdam, the Netherlands.

\section{References}

1. Yau, J. W., Teoh, H., Verma, S. Endothelial cell control of thrombosis. BMC Cardiovascular Disorders. 15, 130 (2015).

2. Pearson, J. D. Endothelial cell function and thrombosis. Best Practice \& Research: Clinical Haematology. 12 (3), $329-341$ (1999).

3. Bochenek, M. L., Schafer, K. Role of Endothelial Cells in Acute and Chronic Thrombosis. Hamostaseologie. 39 (2), 128-139 (2019).

4. Swieringa, F. et al. Platelet Control of Fibrin Distribution and Microelasticity in Thrombus Formation Under Flow. Arteriosclerosis, Thrombosis, and Vascular Biology. 36 (4), 692-699 (2016).

5. Macfarlane, R. G. An Enzyme Cascade in the Blood Clotting Mechanism, and Its Function as a Biochemical Amplifier. Nature. 202, 498-499 (1964).

6. Versteeg, H. H., Heemskerk, J. W., Levi, M., Reitsma, P. H. New fundamentals in hemostasis. Physiological Reviews. 93 (1), $327-358$ (2013).

7. Opneja, A., Kapoor, S., Stavrou, E. X. Contribution of platelets, the coagulation and fibrinolytic systems to cutaneous wound healing. Thrombosis Research. 179, 56-63 (2019).

8. Tabas, I., Garcia-Cardena, G., Owens, G. K. Recent insights into the cellular biology of atherosclerosis. Journal of Cell Biology. 209 (1), 13-22 (2015).

9. Karino, T., Motomiya, M. Flow through a venous valve and its implication for thrombus formation. Thrombosis Research. 36 (3), 245-257 (1984).

10. ISTH Steering Committee for World Thrombosis Day. Thrombosis: a major contributor to global disease burden. Thrombosis Research. 134 (5), 931-938 (2014).

11. Witkin, A. S. Acute and chronic pulmonary embolism: the role of the pulmonary embolism response team. Current Opinion in Cardiology. 32 (6), 672-678 (2017).

12. Kim, N. H. et al. Chronic thromboembolic pulmonary hypertension. European Respiratory Journal. 53 (1), 1801915 (2019).

13. Zhang, M., Zhang, Y., Pang, W., Zhai, Z., Wang, C. Circulating biomarkers in chronic thromboembolic pulmonary hypertension. Pulmonary Circulation. 9 (2), 2045894019844480 (2019).

14. Giri, J. et al. Interventional Therapies for Acute Pulmonary Embolism: Current Status and Principles for the Development of Novel Evidence: A Scientific Statement From the American Heart Association. Circulation. 140 (20), e774-e801 (2019).

15. Sharma, S., Lang, I. M. Current understanding of the pathophysiology of chronic thromboembolic pulmonary hypertension. Thrombosis Research. 164, 136-144 (2018).

16. Eisenberg, P. R. et al. Fibrinopeptide A levels indicative of pulmonary vascular thrombosis in patients with primary pulmonary hypertension. Circulation. 82 (3), 841-847 (1990).

17. Bochenek, M. L. et al. From thrombosis to fibrosis in chronic thromboembolic pulmonary hypertension. Thrombosis and Haemostasis. 117 (4), 769-783 (2017).

18. Nagy, M., Heemskerk, J. W. M., Swieringa, F. Use of microfluidics to assess the platelet-based control of coagulation. Platelets. 28 (5), 441-448 (2017).

19. Barendrecht, A. D. et al. Live-cell Imaging of Platelet Degranulation and Secretion Under Flow. Journal of Visualized Experiments. (125), e55658 (2017)

20. Vajen, T. et al. Laminar Flow-based Assays to Investigate Leukocyte Recruitment on Cultured Vascular Cells and Adherent Platelets. Journal of Visualized Experiments. (134), e57009 (2018). 
21. Michels, A., Swystun, L. L., Mewburn, J., Albanez, S., Lillicrap, D. Investigating von Willebrand Factor Pathophysiology Using a Flow Chamber Model of von Willebrand Factor-platelet String Formation. Journal of Visualized Experiments. (126), e55917 (2017).

22. Smeets, M. W. J., Mourik, M. J., Niessen, H. W. M., Hordijk, P. L. Stasis Promotes Erythrocyte Adhesion to von Willebrand Factor. Arteriosclerosis, Thrombosis, and Vascular Biology. 37 (9), 1618-1627 (2017).

23. Aird, W. C. Endothelial cell heterogeneity. Cold Spring Harbor Perspectives in Medicine. 2 (1), a006429 (2012).

24. Aitsebaomo, J., Portbury, A. L., Schisler, J. C., Patterson, C. Brothers and sisters: molecular insights into arterial-venous heterogeneity. Circulation Research. 103 (9), 929-939 (2008).

25. Szulcek, R. et al. Delayed Microvascular Shear Adaptation in Pulmonary Arterial Hypertension. Role of Platelet Endothelial Cell Adhesion Molecule-1 Cleavage. American Journal of Respiratory and Critical Care Medicine. 193 (12), 1410-1420 (2016).

26. Casa, L. D., Deaton, D. H., Ku, D. N. Role of high shear rate in thrombosis. Journal of Vascular Surgery. 61 (4), 1068-1080 (2015).

27. Jain, A. et al. Assessment of whole blood thrombosis in a microfluidic device lined by fixed human endothelium. Biomedical Microdevices. 18 (4), 73 (2016).

28. van Nieuw Amerongen, G. P., Draijer, R., Vermeer, M. A., van Hinsbergh, V. W. Transient and prolonged increase in endothelial permeability induced by histamine and thrombin: role of protein kinases, calcium, and RhoA. Circulation Research. 83 (11), 1115-1123 (1998).

29. Smits, J. et al. Blood Outgrowth and Proliferation of Endothelial Colony Forming Cells are Related to Markers of Disease Severity in Patients with Pulmonary Arterial Hypertension. International Journal of Molecular Sciences. 19 (12), E3763 (2018).

30. Tobias, M. D., Wambold, D., Pilla, M. A., Greer, F. Differential effects of serial hemodilution with hydroxyethyl starch, albumin, and $0.9 \%$ saline on whole blood coagulation. Journal of Clinical Anesthesia. 10 (5), 366-371 (1998).

31. Jain, A. et al. Primary Human Lung Alveolus-on-a-chip Model of Intravascular Thrombosis for Assessment of Therapeutics. Clinical Pharmacology \& Therapeutics. 103 (2), 332-340 (2018).

32. Veiseh, M., Veiseh, O., Martin, M. C., Asphahani, F., Zhang, M. Short peptides enhance single cell adhesion and viability on microarrays. Langmuir-ACS. 23 (8), $4472-4479$ (2007).

33. Kuo, C. W., Chueh, D. Y., Chen, P. Investigation of size-dependent cell adhesion on nanostructured interfaces. Journal of Nanobiotechnology. 12, 54 (2014).

34. McCormack, J. J., Lopes da Silva, M., Ferraro, F., Patella, F., Cutler, D. F. Weibel-Palade bodies at a glance. Journal of Cell Science. 130 (21), 3611-3617 (2017).

35. Guilarte, M., Sala-Cunill, A., Luengo, O., Labrador-Horrillo, M., Cardona, V. The Mast Cell, Contact, and Coagulation System Connection in Anaphylaxis. Frontiers in Immunology. 8, 846 (2017).

36. Westein, E. et al. Atherosclerotic geometries exacerbate pathological thrombus formation poststenosis in a von Willebrand factor-dependent manner. Proceedings of the National Academy of Sciences. 110 (4), 1357-1362 (2013).

37. Huveneers, S., et al. Vinculin associates with endothelial VE-cadherin junctions to control force-dependent remodeling. Journal of Cell Biology. 196 (5), 641-652 (2012).

38. Szulcek, R., Bogaard, H. J., van Nieuw Amerongen, G. P. Electric cell-substrate impedance sensing for the quantification of endothelial proliferation, barrier function, and motility. Journal of Visualized Experiments. (85), e51300 (2014).

39. Yamasaki, F. et al. Association between arterial stiffness and platelet activation. Journal of Human Hypertension. 19 (7), $527-533$ (2005).

40. Tsai, M. et al. In vitro modeling of the microvascular occlusion and thrombosis that occur in hematologic diseases using microfluidic technology. Journal of Clinical Investigation. 122 (1), 408-418 (2012).

41. Zheng, Y. et al. In vitro microvessels for the study of angiogenesis and thrombosis. Proceedings of the National Academy of Sciences. 109 (24), 9342-9347 (2012).

42. Myers, D. R. et al. Endothelialized microfluidics for studying microvascular interactions in hematologic diseases. Journal of Visualized Experiments. (64), e3958 (2012). 